\title{
ICT BONE Views on the Network of the Future: The Role of Optical Networking
}

\author{
Christina (Tanya) Politi ${ }^{* 1}$, Anna Tzanakaki ${ }^{2}$, Michael O'Mahony ${ }^{3}$, Kostas Katrinis ${ }^{2}$, Peter Van Daele ${ }^{4}$, \\ Mario Pickavet $^{4}$, Dimitra Simeonidou ${ }^{3}$, Alexandros Stavdas ${ }^{1}$, Gerald Franzl ${ }^{5}$, John Mitchell ${ }^{6}$, \\ Piero Castoldi $^{7}$, Salvatore Spadaro ${ }^{8}$, Franco Callegatti ${ }^{9}$, Periklis Petropoulos ${ }^{10}$, \\ Kyriakos Vlachos ${ }^{11}$, Achille Pattavina ${ }^{12}$ \\ *1 University of Peloponnese, Department of Telecommunications Science and Technology \\ Tripolis, Terma Karaiskaki, 22100, Greece, Tel: (+30)2710372222, Fax: (+30) 2710372222 \\ e-mail:tpoliti@uop.gr \\ ${ }^{2}$ Athens Information Technology, PO Box 68, 19.5 km, Markopoulo Avenue, Peania 19002, \\ Athens, Greece \\ ${ }^{3}$ University of Essex, Wivenhoe Park, CO43SQ, UK \\ ${ }^{4}$ IBBT, Ghent University, Belgium \\ ${ }^{5}$ Vienna University of Technology, Favoritenstr. 9/388, 1040 Vienna, Austria \\ ${ }^{6}$ Department of Electronic and Electrical Engineering \\ UCL, Torrington Place, London, WC1E 7JE, UK \\ ${ }^{7}$ Scuola Superiore Sant'Anna, Via Moruzzi 1, 56124 Pisa, Italy \\ ${ }^{8}$ Universitat Politècnica de Catalunya, Barcelona, Spain \\ ${ }^{9}$ DEIS University of Bologna, Via Venezia 52, 47023 Cesena, Italy \\ ${ }^{10}$ Optoelectronics Research Centre, University of Southampton, Southampton, SO17 1 BJ, UK \\ ${ }^{11}$ Computer Engineering and Informatics Department \& Research Academic Computer \\ Technology Institute, University of Patras, Rio, Greece \\ ${ }^{12}$ Dept. of Electronics and Information, Politecnico di Milano, Italy
}

\begin{abstract}
Part of the activities of the ICT EU Network of Excellence "Building the future Optical Network in Europe" (BONE) project is the development of the European Roadmap on Optical Networking in the context of the "Network of the Future". This paper presents a summary of the views of the BONE consortium as they were developed in the framework of the Roadmap activity. The methodology followed in the development of the BONE Roadmap was firstly to identify and analyse the status of broadband access penetration as well as the availability of specific network and IT technologies in various European countries. The data collected were then processed and translated into the traffic volume (distributed across different European countries) that a PanEuropean network would need to support. This information is then used as the basis of a WDM optical network dimensioning exercise performed with the aim of identifying the scale of the required optical network. In addition, based on estimations regarding the evolution of broadband access technologies and the degree of broadband penetration and network services and applications, extrapolations regarding the growth of traffic to be supported by the European Network of the Future were made in a systematic manner. These were also used to dimension and understand the requirements of the European Network of the Future. This paper presents the relevant results and also provides a discussion on the BONE consortium views on the Network of the Future from an architectural and technology perspective and an outlook of the relevant key research challenges and possible solutions.
\end{abstract}

Keywords: optical networks, roadmap, ICT BONE, future optical networking.

\section{INTRODUCTION}

Today's backbone networks already rely on optical technology to provide the capacity required to serve the current broadband access penetration. Broadband access technology worldwide moves towards a number of FTTx variants in order to support the growing demand for new services and associated applications. As far as core networks are concerned it is the combined effect of the access rate growth and bandwidth demanding service availability and popularity that dictates the requirements for this part of the network. The ICT EU Network of Excellence "Building the future Optical Network in Europe" (BONE) is a EU funded project that looks into the future and supports the final "Network of the Future" through education \& training, research tools $\&$ testlabs on new technologies \& architectures. A part of these activities is to consolidate views and develop further the European Roadmap on Optical Networking in the context of the "Network of the Future" by solidifying the previously developed views of the e-photon/ONe+ Network of Excellence. The aims of the Roadmap can be summarised as follows:

- To understand the situation in Europe, based on the wide expertise and views of the BONE partners and representatives, regarding availability of specific network and IT technologies across the different areas of Europe. 
- To develop a realistic view on how network services and applications are evolving and what requirements this may impose on the Network of the Future.

- To extrapolate these requirements in order to translate these to a Pan-European NetworkTraffic Matrix

- To develop a consolidated view on how optical networking can better help meet those requirements

- To identify the missing areas of research needed to support this view of the Network of the Future

- To set out the issues and solutions in a way that will provide options to those people who have the responsibility to develop and implement future networks, network technologies and define areas for further research activity.

\section{BONE ROADMAP METHODOLOGY}

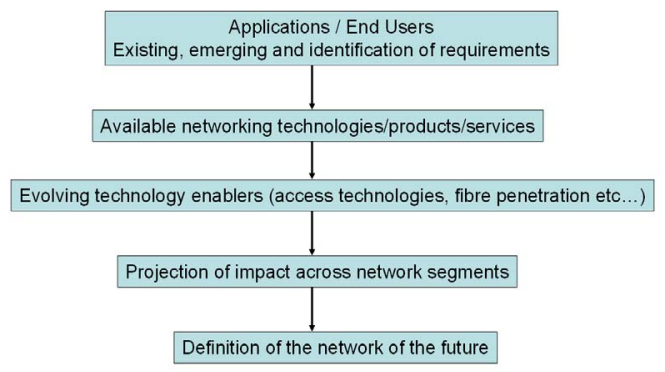

Figure 1. ICT BONE Roadmap Methodology [1].

The methodology followed in the roadmap development is illustrated in Fig. 1. Specifically, information regarding existing and emerging applications and services, broadband penetration, existing and upcoming access technologies was collected for various European countries through a detailed survey performed among BONE partners [1]. The output of this survey addressed, amongst others, the definition of services \& bitrates for residential customers and large enterprises today, in the near future $(\sim 5$ years $)$ and in the future $(>10$ years from now). Special emphasis was paid on the evolving applications and new digital services and the impact that they may have on the network. Definition of the future services and the bitrates of these new services, combined with the access rates give an indication of the growing requirements of the users and access requirements in bandwidth per user according to these predictions are shown in Fig. 2. It is evident that private/household related services are becoming bandwidth demanding and that large enterprises now comprise different industrial sectors from hospitals and campuses and museums to broadcasting industry. The specific applications impose stringent requirements not only on the bandwidth but also the quality of the service, the service delivery time and the level of flexibility that they may require.
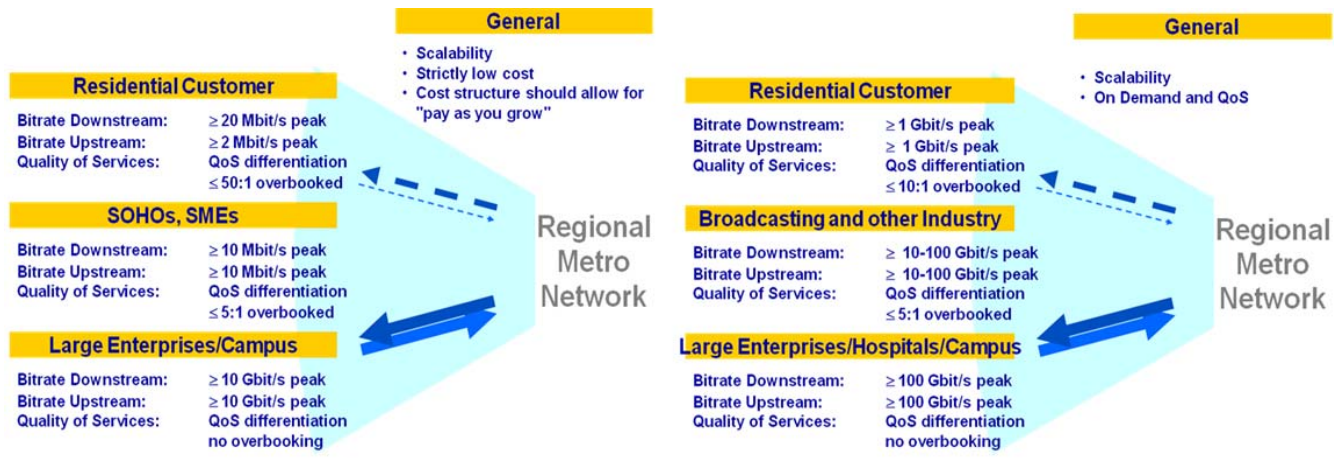

Figure 2. Services \& Bitrates for Large Enterprises today and in the long term Future [1].

In order to evaluate the impact of different technologies and access rates in the core network, the European diversity was considered. This is because the European population distribution and population density plays an important role in the deployment of different new services or technologies, as a critical user mass has to be guaranteed for service providers and network operators to proceed with the economic deployment of new technology. Meanwhile countries in Europe were classified as 'fast' or 'slow' in terms of their deployment on fibre. This is based on among others things, their current technology deployment according to the survey and the business model applied for these deployments; for example public funding may accelerate specific technology uptake in certain regions. The output of this specific part of the Roadmap produced a realistic traffic matrix, which was used as a base for the roadmap network dimensioning exercise. This dimensioning study provided realistic predictions, with regards to capacity requirements, in the network of the future that were in turn used to identify limitations in todays networks while recognising suitable architectural approaches and technology solutions optimised for the network of the future. 


\section{IMPACT OF FUTURE ACCESS TECHNOLOGIES}

Based on the per-country input data, and taking into consideration the parameters described above, an analysis, the details of which can be found in [2], was performed to produce the traffic generated/terminated and supported by the various European countries under consideration. Each country has one point of presence in the Pan-European network and we assumed that $20 \%$ of the traffic generated within each country is fed into this network. The projection of the dimensions of the European network to the mid- and long-term future is performed through solving an instance of the network dimensioning problem. In this version of the problem, the geographical location of nodes is given together with the set of trenched physical links (ducts) connecting neighbouring nodes. The output of the dimensioning process is the optimal number of fibres per link and wavelengths per fibre that need to be installed to serve the input traffic matrix at minimum cost, as well as the optimal dimensions of optical switches required to transparently route the input traffic (Fig. 3).
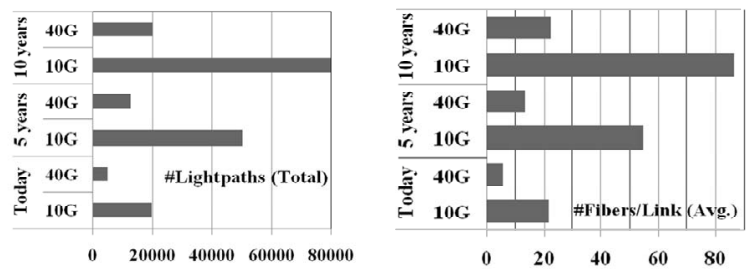

Figure 3. European optical backbone dimensions and Number of fibres per link.

\section{ICT BONE VIEW ON THE NETWORK OF THE FUTURE}

In order to facilitate the penetration of new services and applications the access bandwidth rates should be upgraded accordingly. This will inevitably affect Metropolitan area networks as in this step of the network evolution they may emerge as the main bottleneck with regards to network upgradability and efficient end-to-end service delivery. As far as the core network is concerned important changes should be proposed in order to address the scalability, power efficiency, flexibility and reconfiguration challenges of today's networks. In the last part of this exercise we aim at understanding the limitations of current technologies considering the penetration of new services and the expected access rate growth predicted. In this context we present the views of the BONE consortium on the different network segments of the network of the future.

\subsection{Long Term Vision for Metro/Access Network}
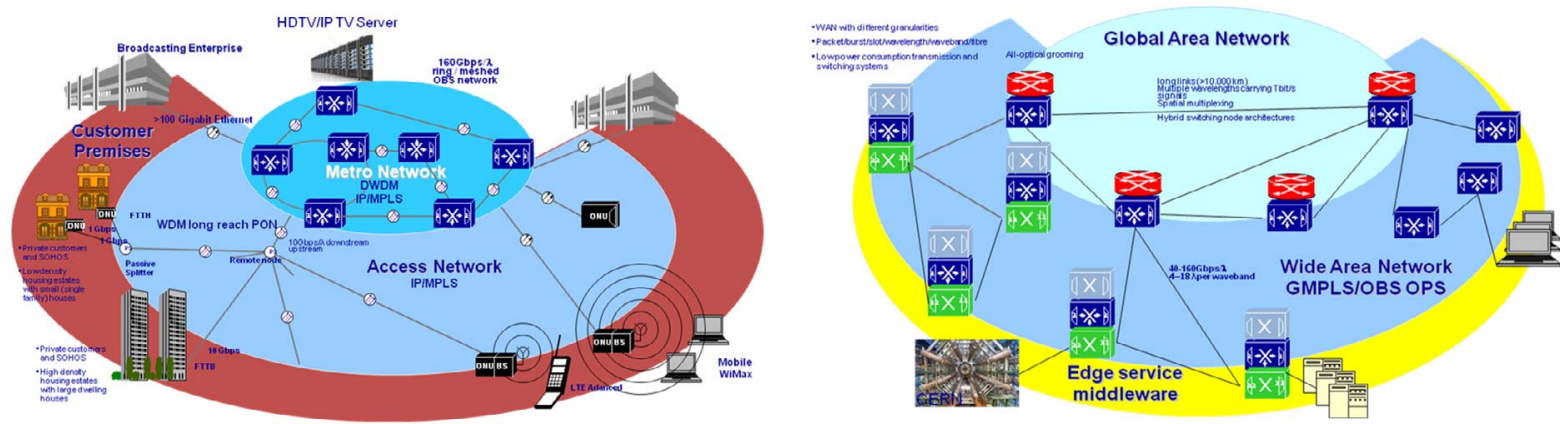

Figure 4. Term Vision for Metro/Access and GAN/WAN Networks.

Regarding the long term vision ( $>10$ years), as shown in the figure above, fiber is expected to be the dominant transmission medium in the access domain for most European countries. Typically the fiber is expected to reach the building for all kind of household and business customers. Within the buildings wireless will play the dominant role for effective bitrates spanning from 10s to $100 \mathrm{Mbps}$. In large business and enterprise buildings fiber will typically be used for very high speed access (100 GEthernet). Point-to-point links and meshed networks will connect large enterprise buildings (FTTB) and also large buildings for private households/customers directly to the metro network. PONs will be further developed concentrating traffic originating from small to medium size buildings for private customers, SOHOs and small to medium businesses and a lot of densely distributed radio base stations. ICT BONE foresees a different FTTx scenario to overcome the limitations of medium term TDMA PONs, which are among others: contended bandwidth, security, flexibility for TDM based protocols and upgradability. Specifically various long reach WDM PON architectures that are seen as a long term solution are discussed. The immense transport capacity of future long reach WDM PONs introduces the need for advance component technology.

As far as the Metro segment is concerned, there is a long debate regarding mesh and ring topologies. Mesh networks outperform rings because of their advantages regarding flexibility, capacity, efficiency, scalability and 
survivability; however, the control simplicity of ring topology and the availability of well established protocols make them a very appealing choice. The compatibility of the proposed solution with optical access technology and core networks for end-to-end service delivery will play an important role in this choice. Metro networks can support large numbers of high speed wavelength channels considering that the transmission distances they have to support are limited and ultra high-speed is possible exceeding e.g. $160 \mathrm{~Gb} / \mathrm{sec}$. Addressing the nature of the input traffic, metro networks are likely to employ optical burst/packet switching, hence offering improved bandwidth efficiency and tolerance to the burstiness of the input traffic. However, it is not anticipated that these solutions will support very high data rates. It is also likely that in this time period wide area networks (WANs) will comprise a small number of high throughput nodes interconnected by high capacity links.

\subsection{Long Term Vision on Global Area Networks and Wide Area Networks (GAN/WAN)}

The long term ICT BONE vision on global area networks (GANs) and WANs is illustrated in Fig. 4. In order to overcome the limitations of current networks, BONE focuses on the different network planes (data, control and service planes) and foresees different technological evolutions per plane that can drive this scenario forward.

As discussed above in case of the WAN, examined through the PanEuropean Network case study, it is clear that significant traffic growth is expected. This is directly reflected on network capacity growth introducing several data plane challenges. A solution that can be proposed to deal with the bandwidth efficiency and granularity requirements of such a network would be a multi-granularity transport supporting switching granularities spanning from sub-wavelength to wavelength bands or even fibers while facilitating node consolidation and traffic grooming. Regarding capacity the single channel bit rate of the WAN will exceed $40 \mathrm{Gbit} / \mathrm{s}$, while using more sophisticated modulation formats and coding schemes the spectral efficiency will increase to values above $1 \mathrm{bit} / \mathrm{s} / \mathrm{Hz}$ in the long haul links. However, these involve advanced technology deployment and the need for standardisation. A very important issue that is arising and will affect the long term evolution of WANs, is the overall network power consumption. Relevant work is performed in BONE with the aim to facilitate energy efficiency through all the different network planes.

Furthermore ICT BONE envisages the introduction of a Service Plane that will be facilitated by an Edge Service Middleware and will obtain the external coordination needed by the UNI/NNI implementations. The Control Plane will incorporate cross-layer optimization mechanisms through suitable extensions of existing routing algorithms to take into consideration physical layer performance issues through e.g. impairment aware routing. In this context GMPLS will be able to facilitate network performance optimization as well as optimal use of available equipment including regenerators, to minimize physical layer impairments in lightpaths and extend the transparency domain as far as possible. In addition, the project is aiming at overcoming limitations such as lack of a multi-domain interoperability framework, developing extensions of the GMPLS paradigm for hybrid OCS/OBS network scenarios, strategies for combating weak IP and WDM integration, and also mechanisms for efficient protection and failure recovery in dynamic optical networks. All these are issues that have been identified to be of major importance in future networks.

\section{ACKNOWLEDGEMENTS}

This work was performed under the support of the BONE-project ("Building the Future Optical Network in Europe"), a Network of Excellence funded by the European Commission in the 7th ICT- Framework Program.

\section{REFERENCES}

[1] http://www.ict-bone.eu/portal/landing_pages/index.html .

[2] T. Politi et al., "2nd report on dissemination activities including updated roadmap", BONE, http://www.ictbone.eu/portal/landing_pages/bone_deliverables/bone_216863_-_d01-4___report_y2.pdf.

[3] B. Mukherjee, "Optical WDM Networks", Springer Verlag, ISBN- $\overline{-}-978-0-\overline{387}-29055-3,2006$. 